\title{
GW23-e1359 DIFFERENT PATTERNS OF AORTIC STIFFNESS AND VASODILATION CAUSED BY $\beta$-BLOCKERS IN SPONTANEOUSLY HYPERTENSIVE RATS
}

doi:10.1136/heartjnl-2012-302920b.38

Gao pingjin Zhouwugang. Departement of Hypertension, RuiJin Hospital, Shanghai Key Lab of Vascular Biology, Shanghai Institute of Hypertension

Objectives This study aims to investigate different patterns of aortic stiffness and vasodilation in spontaneously hypertensive rats treated with $\beta$-blockers, with or without vasodilatory properties.

Methods Spontaneously hypertensive rats (SHRs) were treated with metoprolol $\left(200 \mathrm{mg} \mathrm{kg}^{-1} \mathrm{~d}^{-1}\right)$, arotinolol $\left(30 \mathrm{mg} \mathrm{kg}^{-1} \mathrm{~d}^{-1}\right)$, and nebivolol (15 $\mathrm{mg} \mathrm{kg}^{-1} \mathrm{~d}^{-1}$ ) for 8 weeks. Age-matched WistarKyoto rats and untreated SHRs were used as controls. Central arterial pressure and pulse wave velocity were evaluated via catheter pressure transducers. The rings of rat aorta, and renal and mesenteric arteries were evaluated by isometric force recording. Collagen was assessed by immunohistochemistry, while endothelial nitric oxide synthase (eNOS) and eNOS phosphorylation (p-eNOS) were analysed by western blotting. Nitric oxide and reactive oxygen species were measured in human aortic endothelial cells by using fluorescent probes.

Results Data showed that nebivolol and arotinolol, rather than metoprolol, markedly decreased central arterial pressure and pulse wave velocity at week 8 , and compared to metoprolol, both nebivolol and arotinolol obviously increased vasorelaxation, aortic vasorelaxation to acetylcholine and p-eNOS/eNOS, and the effects of nebivolol were more pronounced. Besides, aortic collagen depositions in the nebivolol and arotinolol groups were reduced compared with those in the metoprolol group or untreated SHRs. Nebivolol and arotinolol produced more nitric oxide and had higher oxygen radical-scavenging capacities compared to metoprolol in the endothelial cells. Intriguingly, a potassium channel inhibitor (4-aminopyridine) caused a significant reduction in artinolol-induced vasorelaxation, but not nebivolol.

Conclusions These findings suggested that vasodilating $\beta$-blockers with nitric oxide production may cause increased reduction of arterial stiffness via different mechanisms. 